\title{
Malignant Pancreatic Vipoma
}

National Cancer Institute

\section{Source}

National Cancer Institute. Malignant Pancreatic Vipoma. NCI Thesaurus. Code C67461.

A pancreatic neuroendocrine tumor producing vasoactive intestinal peptide (VIP). It is associated with watery diarrhea, hypokalemia, and hypochlorhydria or achlorhydria. It displays vascular invasion and metastasizes to other anatomic sites. One third of cases are metastatic at the time of diagnosis. 